\title{
Mesoscopic fluctuations of Coulomb drag between quasiballistic one-dimensional wires
}

\author{
Mortensen, Asger; Flensberg, Karsten; Jauho, Antti-Pekka
}

Published in:

Physical Review B Condensed Matter

Link to article, DOI:

10.1103/PhysRevB.65.085317

Publication date:

2002

Document Version

Publisher's PDF, also known as Version of record

Link back to DTU Orbit

Citation (APA):

Mortensen, A., Flensberg, K., \& Jauho, A-P. (2002). Mesoscopic fluctuations of Coulomb drag between quasiballistic one-dimensional wires. Physical Review B Condensed Matter, 65(8), 085317.

https://doi.org/10.1103/PhysRevB.65.085317

\section{General rights}

Copyright and moral rights for the publications made accessible in the public portal are retained by the authors and/or other copyright owners and it is a condition of accessing publications that users recognise and abide by the legal requirements associated with these rights.

- Users may download and print one copy of any publication from the public portal for the purpose of private study or research.

- You may not further distribute the material or use it for any profit-making activity or commercial gain

- You may freely distribute the URL identifying the publication in the public portal 


\title{
Mesoscopic fluctuations of Coulomb drag between quasiballistic one-dimensional wires
}

\author{
Niels Asger Mortensen \\ Mikroelektronik Centret, Technical University of Denmark, Orsteds Plads, Bld. 345 east, DK-2800 Kgs. Lyngby, Denmark, \\ Orsted Laboratory, Niels Bohr Institute for APG, Universitetsparken 5, DK-2100 Copenhagen , Denmark, and \\ Instituut-Lorentz, Universiteit Leiden, P.O. Box 9506, 2300 RA Leiden, The Netherlands \\ Karsten Flensberg \\ Orsted Laboratory, Niels Bohr Institute for APG, Universitetsparken 5, DK-2100 Copenhagen Ф, Denmark \\ Antti-Pekka Jauho \\ Mikroelektronik Centret, Technical University of Denmark, Drsteds Plads, Bld. 345 east, DK-2800 Kgs. Lyngby, Denmark \\ (Received 17 August 2001; published 7 February 2002)
}

\begin{abstract}
Quasiballistic one-dimensional quantum wires are known to have a conductance of the order of $2 e^{2} / h$, with small sample-to-sample fluctuations. We present a study of the transconductance $G_{12}$ of two Coulomb-coupled quasiballistic wires; i.e., we consider the Coulomb drag geometry. We show that the fluctuations in $G_{12}$ differ dramatically from those of the diagonal conductance $G_{i i}$ : the fluctuations are large and can even exceed the mean value, thus implying a possible reversal of the induced drag current. We report extensive numerical simulations elucidating the fluctuations for both correlated and uncorrelated disorder. We also present analytic arguments, which fully account for the trends observed numerically.
\end{abstract}

DOI: 10.1103/PhysRevB.65.085317

PACS number(s): 73.23.-b, 73.50.-h, 73.61.-r, 85.35.Be

\section{INTRODUCTION}

Recent advances in nanotechnology have made transport studies of quantum wires an active field of study. Several fabrication routes have become available: gated twodimensional (2D) electron gases, cleaved layer overgrowth techniques, grooved high-index surfaces, or even nanotubes. It is quite feasible that in future's nanoelectronic components several quantum wires are very closely spaced (spacing $d$ is of the order of the inverse screening wave vector), and hence the stray fields due to moving charges in one quantum wire affect the motion of charges in the neighboring wires. Analogous effects in coupled quantum wells have been intensively studied for the last ten years (for a review, see Ref. 1) and have become known as Coulomb drag. While only very few experimental studies of coupled quantum wires have been reported so far (however, see Refs. 2 and 3), we believe that more experiments will become available in the near future.

Coulomb drag of mesoscopic structures has been addressed theoretically in the case of 1D systems both within the Boltzmann equation approach ${ }^{4,5}$ and for Luttinger liquids with strong interwire interactions. ${ }^{6-11}$ For the latter case, the interesting possibility of a regime with almost identical currents in the two wires has been predicted ${ }^{6-9}$ and also interesting effects for drag between carbon nanotubes have been found. ${ }^{9,10}$

Disorder will inevitably be present in all real samples. The study of fluctuation phenomena in mesoscopic systems is a mature field and has tremendously increased our understanding of the basic physics governing electronic transport in solids. The observation and explanation of universal conductance fluctuations ${ }^{12}$ is one of the central achievements in this field. It is then natural to ask, are similar phenomena present in the case of Coulomb coupled systems? In other words, what are the fluctuation properties of the transconductance?

Quite recently the study of fluctuations of the Coulomb drag was initiated by Narozhny and Aleiner. ${ }^{13}$ These ideas were used by us to study various properties of Coulomb drag in systems smaller than the Thouless energy. ${ }^{14,15}$ They provided answers to some aspects to the question phrased above. In particular, in Ref. 13 it was shown that the fluctuations will be pronounced for temperatures smaller than the Thouless energy. In contrast to the universal conductance fluctuations $^{12}$ of the Landauer conductance $G_{i i}$ the fluctuations of $G_{21}$ are, however, nonuniversal and depend on temperature and various system parameters.

In this work we focus on coupled quasiballistic 1D wires (see Fig. 1) and show that even weak disorder can change the ballistic properties drastically and give rise to new interest-

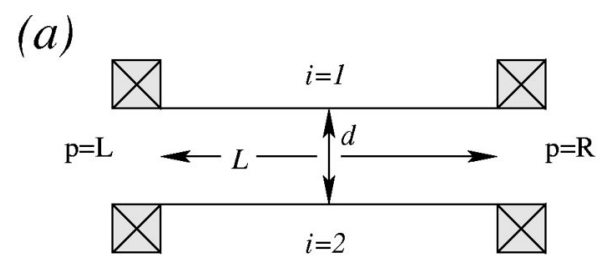

(b)

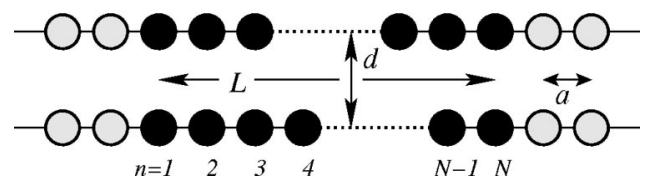

FIG. 1. (a) Geometry of the two coupled 1D wires of length $L$ and separation $d$. (b) Lattice model of the two 1D wires. Here, denote the lattice points of the wires (where Coulomb interaction $U_{12}$ is effective) and $\bigcirc$ denote those belonging to the ideal leads. 
ing phenomena. The paper also gives a more detailed derivation of some technical results used in our recent Letter. ${ }^{14}$

We consider temperatures smaller than $\hbar v_{F} / L$ so that intrawire interactions are effectively given by the Fermi liquid reservoirs and where the screening can be considered to be static. In this regime it is well known from calculation of drag between clean Luttinger liquids that the intrawire interactions have no pronounced effect. ${ }^{6,7}$ For the interwire interaction we consider the physical relevant regime with longrange interaction where $U_{12}(q=0) \gg U_{12}\left(q=2 k_{F}\right)$.

Starting from the Kubo formula we derive an expression for the transconductance $G_{21}$ similar to previous results 16,17 but relaxing the assumption of translational invariance. ${ }^{14} \mathrm{We}$ formulate the problem with the help of the spectral function and subsequently map it onto a tight-binding-like model suitable for a computer implementation. Our formalism allows us to treat wires with electrons propagating in arbitrary potentials. The fluctuations in drag between disordered wires are studied by numerical ensemble averaging and also analytically with the disorder included perturbatively. We consider two situations: uncorrelated and correlated disorder. For the situation where the disorder potentials of the two wires are mutually uncorrelated, as is usually assumed, ${ }^{13,14,17}$ we find large fluctuations of the order of the mean value and thereby also a possible sign reversal of the drag current. If the two disorder potentials are identical, i.e., mutually correlated, ${ }^{18}$ we get large fluctuations and an enhanced mean value compared to uncorrelated disorder. We also give some results for the intermediate case. The case of fully correlated disorder is in qualitative agreement with recent predictions for $2 \mathrm{D}$ systems ${ }^{18}$ and for the fluctuations we predict an enhancement by a factor of $\sqrt{2}$ which is confirmed numerically.

The obtained distributions are quite robust in the sense that systems with different disorder strength and/or length and separation can be rescaled to fall on the same curve. However, the distributions also depend on the range of the interaction $U_{12}$ and in that sense they are nonuniversal.

The paper is organized as follows: in Sec. II we introduce the formalism and in Sec. III we derive analytic results for quasiballistic wires. Section IV contains our numerical results. Finally, in Sec. V discussions and conclusions are given. Certain technical details can be found in the appendixes.

\section{FORMALISM}

\section{A. General formulation}

Let us consider two 1D wires of length $L$ (shorter than the phase-coherence length $l_{\phi}$ ) parallel to each other with a separation $d$; see Fig. 1. Our aim is to calculate the dc $(\Omega$ $\rightarrow 0$ ) linear-response transconductance $G_{21}=\partial I_{2} / \partial V_{1}$. In the $1 \mathrm{D}$ situation the transconductance is equal to the transconductivity which can be calculated from Kubo formalism ${ }^{19}$ which expresses $G_{21}$ in terms of the retarded current-current correlation function: ${ }^{17}$

$$
\begin{aligned}
G_{21}= & \lim _{\Omega \rightarrow 0} \frac{e^{2}}{\hbar \Omega} \int_{0}^{\infty} d\left(t-t^{\prime}\right) \exp \left[i \Omega\left(t-t^{\prime}\right)\right] \\
& \times\left\langle\left[\hat{J}_{2}\left(x_{2}, t\right), \hat{J}_{1}\left(x_{1}, t^{\prime}\right)\right]\right\rangle .
\end{aligned}
$$

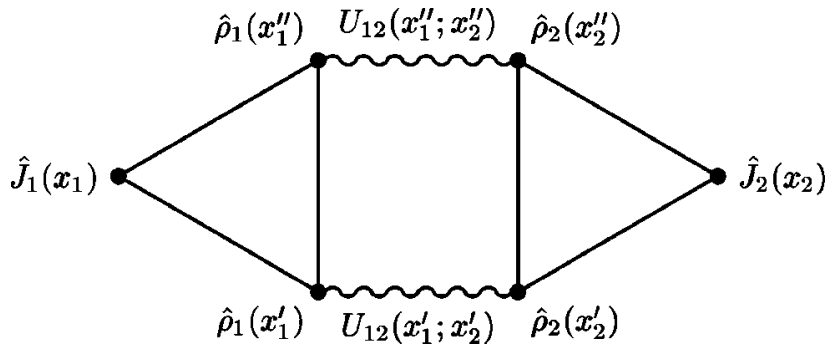

FIG. 2. Diagrammatic representation of the current-current correlation function $\Delta_{1} U_{12} U_{12} \Delta_{2}$; see Eq. (2).

Here, $\hat{J}_{i}$ is the particle current operator of wire $i=1,2$. The brackets indicate the quantum mechanical statistical average. Due to current conservation, $x_{1}$ and $x_{2}$ can be chosen at any position along the wires. ${ }^{20,21}$ Using Matsubara formalism we follow Ref. 17 and calculate $G_{21}$ to second order in the interaction $U_{12}$ between the mesoscopic 1D wires. Intrawire interactions are neglected, except for disorder potential scattering. The expansion of the time-development operator to second order in $U_{12}$ gives rise to a current-current correlation function which can be expressed as a product of two threepoint correlation functions: $\left\langle\hat{\rho}_{1} \hat{\rho}_{1} \hat{J}_{1}\right\rangle U_{12} U_{12}\left\langle\hat{\rho}_{2} \hat{\rho}_{2} \hat{J}_{2}\right\rangle$, where $\hat{\rho}_{i}$ is the particle density operator. The three-point correlation functions are evaluated with the aid of Wick's theorem and after some lengthy, but in principal straightforward calculations, we arrive at

$$
\begin{aligned}
G_{21}= & \frac{e^{2}}{h} \int_{0}^{L} \int_{0}^{L} \int_{0}^{L} \int_{0}^{L} d x_{1}^{\prime} d x_{2}^{\prime} d x_{1}^{\prime \prime} d x_{2}^{\prime \prime} \int_{-\infty}^{\infty} \hbar d \omega \\
& \times \frac{\Delta_{1}\left(\omega, x_{1}^{\prime}, x_{1}^{\prime \prime}\right) U_{12}\left(x_{1}^{\prime} ; x_{2}^{\prime}\right) U_{12}\left(x_{1}^{\prime \prime} ; x_{2}^{\prime \prime}\right) \Delta_{2}\left(\omega, x_{2}^{\prime \prime}, x_{2}^{\prime}\right)}{2 k T \sinh ^{2}(\hbar \omega / 2 k T)},
\end{aligned}
$$

$$
\begin{aligned}
\Delta_{i}\left(\omega ; x^{\prime}, x^{\prime \prime}\right)= & \frac{1}{4 \pi} \frac{\hbar^{2}}{2 m} \int d \varepsilon A_{\varepsilon-\hbar \omega}^{i}\left(x^{\prime \prime}, x^{\prime}\right) \\
& \times\left[A_{\varepsilon}^{i}\left(x, x^{\prime \prime}\right) \partial_{x} A_{\varepsilon}^{i}\left(x^{\prime}, x\right)\right. \\
& \left.-A_{\varepsilon}^{i}\left(x^{\prime}, x\right) \partial_{x} A_{\varepsilon}^{i}\left(x, x^{\prime \prime}\right)\right] \\
& \times\left[n_{F}(\varepsilon-\hbar \omega)-n_{F}(\varepsilon)\right]-(\omega \rightarrow-\omega)^{*}, \\
A_{\varepsilon}^{i}\left(x, x^{\prime}\right) & =i\left[\mathcal{G}_{\varepsilon}^{i}\left(x, x^{\prime}\right)-\left\{\mathcal{G}_{\varepsilon}^{i}\left(x^{\prime}, x\right)\right\}^{*}\right] \\
& =2 \pi \sum_{\alpha}\langle x \mid \alpha\rangle\left\langle\alpha \mid x^{\prime}\right\rangle \delta\left(\varepsilon-\varepsilon_{\alpha}\right) .
\end{aligned}
$$

Here, $A_{\varepsilon}^{i}$ is the spectral function, $\mathcal{G}_{\varepsilon}^{i}$ the retarded Green function, $n_{F}(\varepsilon)$ the Fermi-Dirac distribution function, and $\varepsilon_{\alpha}$ is the eigenvalue of the exact single-particle eigenstate $|\alpha\rangle$ in the uncoupled wires. We furthermore assume spin degeneracy. The current-current correlation function $\Delta_{1} U_{12} U_{12} \Delta_{2}$ is shown schematically in Fig. 2.

Equation (2) generalizes the results of Ref. 17 to broken translational invariance, and Appendix A establishes a connection to the expression employed in our recent Letter. ${ }^{14}$ 


\section{B. Lattice formulation}

Writing the four spatial integrals in Eq. (2) as sums over the functions on a discrete lattice (see Fig. 1), we get

$$
G_{21}=\frac{e^{2}}{h} \int_{-\infty}^{\infty} \hbar d \omega \frac{\operatorname{Tr}\left[U_{12}^{T} \Delta_{1}(\omega) U_{12} \Delta_{2}(\omega)\right]}{2 k T \sinh ^{2}(\hbar \omega / 2 k T)},
$$

where the matrices have elements

$$
\begin{aligned}
& \left\{U_{12}\right\}_{n n^{\prime}}=U_{12}\left(x_{1}^{\prime} \rightarrow n a ; x_{2}^{\prime} \rightarrow n^{\prime} a\right), \\
\left\{\Delta_{i}(\omega)\right\}_{n n^{\prime}}= & \frac{1}{4 \pi} \frac{\hbar^{2}}{2 m a^{2}} \int d \varepsilon\left\{A_{\varepsilon-\hbar \omega}^{i}\right\}_{n^{\prime} n} \\
& \times\left[\left\{A_{\varepsilon}^{i}\right\}_{\tilde{n} n^{\prime}}\left\{A_{\varepsilon}^{i}\right\}_{n, \tilde{n}+1}-\left\{A_{\varepsilon}^{i}\right\}_{n \tilde{n}}\left\{A_{\varepsilon}^{i}\right\}_{\tilde{n}+1, n^{\prime}}\right] \\
& \times\left[n_{F}(\varepsilon-\hbar \omega)-n_{F}(\varepsilon)\right]-(\omega \rightarrow-\omega)^{*}, \\
& \left\{A_{\varepsilon}^{i}\right\}_{n n^{\prime}} \equiv a \times A_{\varepsilon}^{i}\left(x \rightarrow n a, x^{\prime} \rightarrow n^{\prime} a\right),
\end{aligned}
$$

where $n, n^{\prime}=1,2,3, \ldots, N$ label the lattice points and $a$ is the lattice constant; see Fig. 1(b). In matrix notation, $A_{\varepsilon}^{i}=i\left[\mathcal{G}_{\varepsilon}^{i}\right.$ $\left.-\left\{\mathcal{G}_{\varepsilon}^{i}\right\}^{\dagger}\right]$. The derivative $\partial_{x}$ in $\Delta$ has been accounted for by the method of finite differences ${ }^{22}$ and $\tilde{n}$ can be any lattice points $1,2,3, \ldots, N-2, N-1$ due to current conservation. Summing over the first $N-1$ lattice points and dividing by $N-1$ we find

$$
\begin{aligned}
\Delta_{i}(\omega)= & \frac{1}{4 \pi} \frac{\hbar^{2}}{2 m a^{2}} \int d \varepsilon\left\{A_{\varepsilon-\hbar \omega}^{i}\right\}^{T} \otimes\left[A_{\varepsilon}^{i} \Lambda A_{\varepsilon}^{i}\right] \\
& \times\left[n_{F}(\varepsilon-\hbar \omega)-n_{F}(\varepsilon)\right]-(\omega \rightarrow-\omega)^{*},
\end{aligned}
$$

where $\otimes$ denotes an element-by-element multiplication, $\{X$ $\otimes Y\}_{n m}=X_{n m} Y_{n m}$, and the matrix $\Lambda$ has elements

$$
\Lambda_{n n^{\prime}}=\frac{ \pm \delta_{n, n^{\prime} \pm 1}}{N-1}
$$

The next step is to calculate the lattice representation of the retarded Green functions $\mathcal{G}^{1}$ and $\mathcal{G}^{2}$ of the two uncoupled wires. Writing the Laplacian $\partial_{x}^{2}$ in the wire Hamiltonians with the help of finite differences the problem of the uncoupled wires is mapped onto tight-binding-like Hamiltonians $^{22}$

$$
H_{n n^{\prime}}^{(i)}=\left(2 t+\left\{V_{i}\right\}_{n}\right) \delta_{n n^{\prime}}-t \delta_{n, n^{\prime} \pm 1}, \quad t=\hbar^{2} / 2 m a^{2} .
$$

A standard approach based on Dyson's equation then gives the retarded Green functions of the uncoupled wires as an $N \times N$ matrix, ${ }^{22}$

$$
\mathcal{G}_{\varepsilon}^{i}=\left[\varepsilon-H^{i}-\Sigma_{\mathrm{L}}^{i}(\varepsilon)-\Sigma_{\mathrm{R}}^{i}(\varepsilon)\right]^{-1},
$$

where the couplings to lead $p=\mathrm{L}, \mathrm{R}$ are taken care of by the retarded self-energy,

$$
\left\{\Sigma_{p}^{i}(\varepsilon)\right\}_{n n^{\prime}}=-t \exp [i k(\varepsilon) a] \delta_{n, n_{p}} \delta_{n_{p}, n^{\prime}}
$$

with $n_{\mathrm{L}}=1$ and $n_{\mathrm{R}}=N$. The wave vector is related to the energy through the usual cosine dispersion relation with a bandwidth of $4 t$, i.e., $\varepsilon=2 t(1-\cos k a)$.

\section{Low-temperature expansion}

We now consider the low-temperature limit $k T \ll \varepsilon_{F}$ where we can evaluate the spectral functions in Eq. (3) at the Fermi level. Performing the energy integration gives $\Delta_{i} \propto \omega$ and the $\omega$ integration in Eq. (2) can now be done:

$$
\int_{-\infty}^{\infty} \hbar d \omega \frac{(\hbar \omega)^{2}}{2 k T \sinh ^{2}(\hbar \omega / 2 k T)}=\frac{4 \pi^{2}}{3}(k T)^{2},
$$

and we get $G_{21} \propto T^{2}$. Equation (5) now simplifies to

$$
G_{21}=\frac{e^{2}}{h}(k T)^{2} \frac{t^{2}}{3} \operatorname{Tr}\left[U_{12} M_{1} U_{12} M_{2}\right]
$$

where

$$
M_{i}=\operatorname{Re}\left\{\left\{A_{\varepsilon_{F}}^{i}\right\}^{T} \otimes\left[A_{\varepsilon_{F}}^{i} \Lambda A_{\varepsilon_{F}}^{i}\right]\right\} .
$$

Equation (15) forms the basis for all subsequent numerical work.

The Landauer conductance $G_{i i}=\partial I_{i} / \partial V_{i}$ of the individual wires can be expressed in a similar form ${ }^{22}$

$$
G_{i i}=\frac{2 e^{2}}{h} \operatorname{Tr}\left[\Gamma_{\mathrm{L}}^{i}\left(\varepsilon_{F}\right) \mathcal{G}_{\varepsilon_{F}}^{i} \Gamma_{\mathrm{R}}^{i}\left(\varepsilon_{F}\right)\left\{\mathcal{G}_{\varepsilon_{F}}^{i}\right\}^{\dagger}\right],
$$

where the leads are described by

$$
\Gamma_{p}^{i}(\varepsilon)=i\left[\Sigma_{p}^{i}(\varepsilon)-\left\{\Sigma_{p}^{i}(\varepsilon)\right\}^{\dagger}\right] .
$$

The matrix formulation is readily implemented on a computer and the accuracy can be increased simply by having more lattice points $N$ (for a given $L$ ). ${ }^{22}$ The retarded Green function can be obtained either by a direct inversion as indicated in Eq. (12) or by a recursive method; see, e.g., Ref. 23.

Alternatively one can also view Eqs. (15) and (17) as formulas for a tight-binding system where $t$ is a "hopping matrix element" between different orbitals and the local potential $V_{n}+2 t$ is the energy of the orbital localized at site $n$. Finally we note that the form of Eq. (15) is valid for any number of transverse channels [for 2D and 3D systems $\Lambda$ in Eq. (10) should be modified to also contain an "integration" over the transverse direction in order to get the total current] and thus provides a versatile starting point for investigations of drag in different geometries.

\section{Anderson model}

For the study of disordered wires we use the Anderson model with diagonal disorder ${ }^{24}$ where the site potentials $V_{n}^{i}$ of a given wire are statistically independent with each site energy taken from a uniform distribution of width $W$ and zero mean (see also Ref. 25). The backscattering mean free path $l$ can be related to the disorder strength $W$ and in the Born approximation we get (see Appendix B)

$$
l=12 a\left(4 t \varepsilon_{F}-\varepsilon_{F}^{2}\right) / W^{2} .
$$




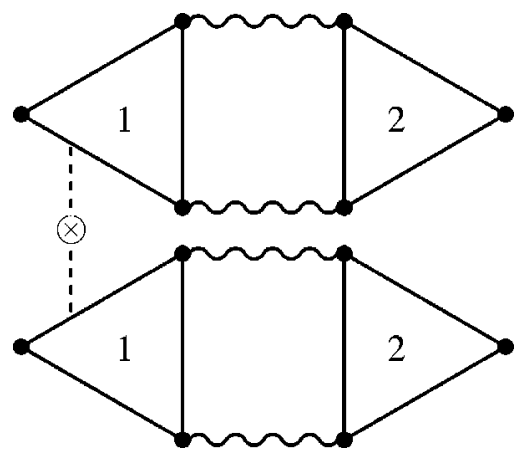

FIG. 3. Example of a first-order connected diagram in the diagrammatic expansion for the fluctuations giving rise to a $\left\langle\left[\delta G_{21}\right]^{2}\right\rangle \propto V^{2} \propto 1 / k_{F} l$ dependence. Due to momentum conservation, the impurity line must carry $q=0$ and the four interaction lines $2 k_{F}$.

We will examine several different ways of realizing the disorder in the two wires. These include a situation where both wires are disordered, but with $V_{1}$ and $V_{2}$ mutually uncorrelated (as in Ref. 14). We also present results for disordered wires where the disorder is fully correlated, i.e., $V_{1}$ $=V_{2}$ (as suggested in Ref. 18).

\section{ANALYTICAL RESULTS}

In this section we consider quasiballistic wires. For weak disorder it is possible to make a perturbation expansion for the fluctuations ${ }^{13} \delta G_{21}=G_{21}-\left\langle G_{21}\right\rangle$ (brackets indicate ensemble averaging).

\section{A. Fluctuations of the order of $V^{2}$}

An example of a lowest-order connected fluctuation diagram is shown in Fig. 3. Due to momentum conservation, the impurity line must carry $q=0$ (corresponding to forward scattering) and the four interaction lines $2 k_{F}$ so that we estimate the magnitude as follows:

$$
\left\langle\left[\delta G_{21}\right]^{2}\right\rangle \propto V^{2}(0) U_{12}^{4}\left(2 k_{F}\right),
$$

where $V(q)$ and $U_{12}(q)$ are the Fourier transforms of the disorder potential and the Coulomb interaction potential, respectively. Though diagrams of order $V^{4}$ are parametrically smaller, we shall see that they give the dominant contribution in the case of long-range Coulomb interaction.

\section{B. Fluctuations of the order of $V^{4}$}

Figure 4 shows examples of connected diagrams of the order of $V^{4}$; now the impurity lines can carry both $q=0$ and $q=2 k_{F}$ corresponding to backscattering. For backscattering in the disorder channel, in the case of uncorrelated (uc) disorder we get the estimates

$$
\begin{gathered}
a:\left\langle\left[\delta G_{21}\right]^{2}\right\rangle_{\mathrm{uc}} \propto V^{4}\left(2 k_{F}\right) U_{12}^{4}\left(2 k_{F}\right), \\
b:\left\langle\left[\delta G_{21}\right]^{2}\right\rangle_{\mathrm{uc}}=0, \\
c:\left\langle\left[\delta G_{21}\right]^{2}\right\rangle_{\mathrm{uc}} \propto V^{4}\left(2 k_{F}\right) U_{12}^{2}(0) U_{12}^{2}\left(2 k_{F}\right),
\end{gathered}
$$

(a)

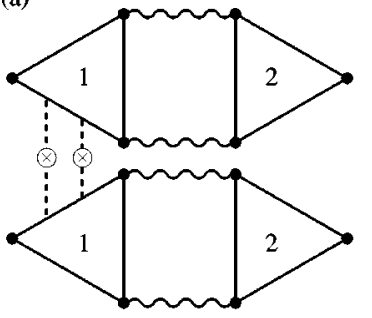

(c)

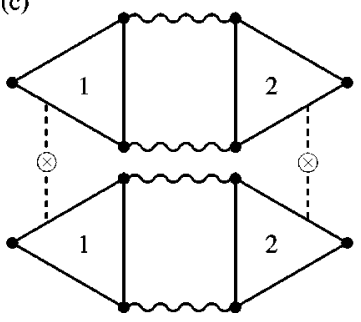

(b)

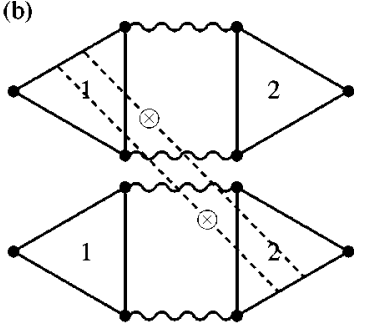

(d)

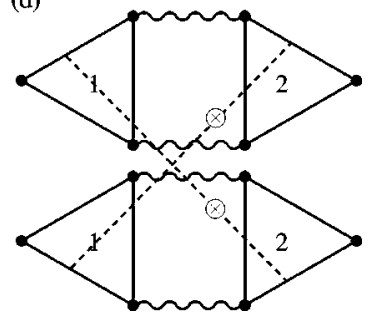

FIG. 4. Examples of second-order connected diagrams in the diagrammatic expansion for the fluctuations giving rise to a $\left\langle\left[\delta G_{21}\right]^{2}\right\rangle \propto V^{4} \propto 1 /\left(k_{F} l\right)^{2}$ dependence. Due to momentum conservation, diagrams with only one impurity line do not contribute to the drag fluctuations. Diagrams (a) and (c) are relevant for both correlated and uncorrelated disorder whereas the diagrams in panels (b) and (d) are relevant for correlated disorder only.

$$
d:\left\langle\left[\delta G_{21}\right]^{2}\right\rangle_{\mathrm{uc}}=0
$$

where to lowest order $V^{2}\left(2 k_{F}\right) \propto 1 / k_{F} l$ so that

$$
\left\langle\left[\delta G_{21}\right]^{2}\right\rangle^{1 / 2} \propto 1 / k_{F} l
$$

For a long-range Coulomb potential $U_{12}(0) \gg U_{12}\left(2 k_{F}\right)$ the dominant contribution comes from diagram $c$ which also dominates parametrically over the contribution to second order; see Eq. (20). Screening mainly affects the small- $q$ limit of $U_{12}(q)$. The mean value $\left\langle G_{21}\right\rangle \propto U_{12}^{2}\left(q=2 k_{F}\right)$ is thus only weakly affected by screening whereas the $V^{4}$ contributions to the fluctuations will be strongly suppressed due to the presence of also $U_{12}^{2}(q=0)$; see Eqs. (21). For sufficiently shortrange interaction the $V^{2}$ contributions will eventually give the dominant contribution [see Eq. (20)], but then the overall magnitude of the fluctuations will also be small compared to the mean value.

For correlated disorder (c) all diagrams $a, b, c$, and $d$ contribute (equally) whereas for uncorrelated disorder only the diagrams $a$ and $c$ are relevant. More generally, for each topologically different diagram contributing in the case of uncorrelated disorder there are two similar diagrams contributing equally in case of correlated disorder. Of course there are also other possible diagrams in the case of correlated disorder but to lowest order in $1 / k_{F} l$ this means that

$$
\begin{aligned}
\frac{\left\langle\left[\delta G_{21}\right]^{2}\right\rangle_{\mathrm{c}}^{1 / 2}}{\left\langle\left[\delta G_{21}\right]^{2}\right\rangle_{\mathrm{uc}}^{1 / 2}} & \simeq \sqrt{\frac{\langle a\rangle+\langle b\rangle+\langle c\rangle+\langle d\rangle}{\langle a\rangle+\langle c\rangle}} \\
& =\sqrt{\frac{2 \times\langle a\rangle+2 \times\langle c\rangle}{\langle a\rangle+\langle c\rangle}}=\sqrt{2},
\end{aligned}
$$


where $\langle a\rangle,\langle b\rangle,\langle c\rangle$, and $\langle d\rangle$ refer symbolically to the diagrams in Fig. 4 averaged over disorder. This simple argument suggests that the fluctuations in the case of correlated disorder will be enhanced by a factor of $\sqrt{2}$ compared to the case of uncorrelated disorder.

We now turn into a quantitative evaluation of the fluctuations. In Appendix D we show how $\Delta_{i}\left(x, x^{\prime}\right)$ can be expressed in terms of scattering states $\psi_{ \pm}(x)$. We introduce a shorthand notation

$$
G_{21}=\mathcal{C} \int U_{12}(x, y) U_{12}\left(x^{\prime} y^{\prime}\right) \Delta_{1}\left(x, x^{\prime}\right) \Delta_{2}\left(y^{\prime}, y\right),
$$

with

$$
\Delta\left(x, x^{\prime}\right)=\operatorname{Im}\left\{\tilde{\rho}_{+-}(x) \tilde{\rho}_{-+}\left(x^{\prime}\right)\right\},
$$

where $\int$ means an integral over all spatial degrees of freedom (in this case $x, x^{\prime}, y$, and $y^{\prime}$ ). Here, $\tilde{\rho}$ are the particle density matrix elements taken in a new basis $\widetilde{\psi}_{ \pm}(x)$ related to the original basis $\psi_{ \pm}(x)$ by a unitary transformation described in Appendix D. The frequency integration has been performed to give the $T^{2}$ dependence and all prefactors are included in $\mathcal{C}$, i.e., $\mathcal{C} \propto\left(e^{2} / h\right)(k T)^{2}$.

Before calculating the prefactor in Eq. (22) in the case of uncorrelated disorder we first consider the transconductance in the case of ideal ballistic wires. Using the free plane waves $\widetilde{\psi}_{ \pm}(x)=\psi_{ \pm}(x)=e^{ \pm i k_{F} x}$ we get

$$
\bar{\Delta}\left(x, x^{\prime}\right)=\operatorname{Im}\left\{e^{-i 2 k_{F} x} e^{i 2 k_{F} x^{\prime}}\right\}=\sin 2 k_{F}\left(x^{\prime}-x\right) .
$$

The electrons in each wire can be either forward scattered (zero momentum) or backscattered $\left(2 k_{F}\right)$ and because of momentum conservation both the interaction lines in the diagram in Fig. 2 carry momentum $2 k_{F}$ (zero momentum from forward scattering does not contribute to drag in this situation) so that

$$
G_{21}(\infty)=\mathcal{C} \frac{1}{2} U_{12}^{2}\left(2 k_{F}\right)
$$

where

$$
U_{12}(q)=\int_{0}^{L} \int_{0}^{L} d x_{1} d x_{2} e^{i q\left(x_{1}-x_{2}\right)} U_{12}\left(x_{1}, x_{2}\right) .
$$

In what follows we often express the results for disordered systems normalized with respect to the ballistic transconductance $G_{21}(\infty)$.

The situation is very different even for weak disorder, and the broken translational invariance allows for transferred momentum others than $2 k_{F}$. For the diagrams in Fig. 4 the impurity lines carry momentum $2 k_{F}$ corresponding to backscattering within the wires (forward scattering will not contribute to the fluctuations). However, the difference between diagrams (a) and (c) is that in (a) the interaction lines in each subdiagram must carry the same momentum whereas in (c) one of them can carry, e.g., zero momentum while the other carries $2 k_{F}$. Now, since $U_{12}(0) \gg U_{12}\left(2 k_{F}\right)$ for long-ranged interaction [typically, $U_{12}(q)$ decays exponentially], diagram (c) gives the major contribution to the fluctuations. This can be tested numerically by noting that diagram (a) is relevant to the case where wire 1 is disordered and wire 2 is either disordered or ballistic whereas diagram (c) is only relevant to the case of both wires being disordered. Indeed, by numerically calculating the fluctuations for a system where one of the wires is ballistic and the other disordered we have found a very dramatic reduction (by more than an order of magnitude) of the fluctuations compared to the case of both wires being disordered.

Considering wires with mutually uncorrelated disorder and writing $\Delta\left(x, x^{\prime}\right)=\bar{\Delta}\left(x, x^{\prime}\right)+\delta \Delta\left(x, x^{\prime}\right)$ (where the second term is the correction to the ballistic limit) the contribution from diagram $(c)$ is

$$
\begin{aligned}
\left\langle\left[\delta G_{21}\right]^{2}\right\rangle_{\mathrm{uc}} \simeq & \mathcal{C}^{2} \int U_{12}(x, y) U_{12}\left(x^{\prime}, y^{\prime}\right) U_{12}(\bar{x}, \bar{y}) \\
& \times U_{12}\left(\bar{x}^{\prime}, \bar{y}^{\prime}\right)\left\langle\delta \Delta_{1}\left(x, x^{\prime}\right) \delta \Delta_{1}\left(\bar{x}, \bar{x}^{\prime}\right)\right\rangle \\
& \times\left\langle\delta \Delta_{2}\left(y^{\prime}, y\right) \delta \Delta_{2}\left(\bar{y}^{\prime}, \bar{y}\right)\right\rangle,
\end{aligned}
$$

and the remaining problem is to calculate the correlation $\left\langle\delta \Delta_{i}\left(x, x^{\prime}\right) \delta \Delta_{i}\left(\bar{x}, \bar{x}^{\prime}\right)\right\rangle$ to lowest order (second order) in the disorder strength. For quasiballistic wires $(\mathcal{T}=1-\mathcal{R} \sim 1)$ Eqs. (D5) and (D6) give

$$
\Delta\left(x, x^{\prime}\right) \approx \operatorname{Im}\left\{\rho_{+-}(x) \rho_{-+}\left(x^{\prime}\right)\right\},
$$

with $\rho_{\alpha \beta}(x)=\psi_{\alpha}^{*}(x) \psi_{\beta}(x)$ in the original basis and formally we thus have that

$$
\delta \Delta\left(x, x^{\prime}\right)=\operatorname{Im}\left\{\delta \rho_{+-}(x) e^{i 2 k_{F} x^{\prime}}+e^{-i 2 k_{F} x} \delta \rho_{+-}^{*}\left(x^{\prime}\right)\right\} .
$$

Using the Lippmann-Schwinger equation ${ }^{26}$ we have to lowest order in the disorder strength that

$$
\psi_{ \pm}(x) \simeq e^{ \pm i k_{F} x}+\int_{0}^{L} d \chi \mathcal{G}_{0}(x, \chi) V(\chi) e^{ \pm i k_{F} \chi}
$$

where $\mathcal{G}_{0}\left(x, x^{\prime}\right)=\left(i \hbar v_{F}\right)^{-1} e^{i k_{F}\left|x^{\prime}-x\right|}$ is the unperturbed retarded Green function. This means that $\rho_{+_{-}}(x)=\rho_{+-}^{0}(x)$ $+u(x)+\nu(x)$ where

$$
\begin{aligned}
& u(x)=\left(i \hbar v_{F}\right)^{-1} \int_{0}^{L} d \chi \operatorname{sign}(x-\chi) V(\chi) \rho_{+-}^{0}(\chi), \\
& \nu(x)=\left(i \hbar v_{F}\right)^{-1} \int_{0}^{L} d \chi \operatorname{sign}(\chi-x) V(\chi) \rho_{+-}^{0}(x) .
\end{aligned}
$$

Here, $\rho_{+-}^{0}(x)=\exp \left(-2 i k_{F} x\right)$. Thus we see that $u$ gives a nonoscillating correction to $\rho_{+-}^{0}(x)$ which is the reason why the Fourier component $U_{12}(0)$ enters the end result instead of $U_{12}\left(2 k_{F}\right)$. This is not the case for $v$ and we can therefore omit it. For the averages over disorder we use $\left\langle V(\chi) V\left(\chi^{\prime}\right)\right\rangle=W_{0}^{2} \delta\left(\chi-\chi^{\prime}\right)$ and neglecting terms oscillating with $4 k_{F}$ we get $\langle u(x) u(\bar{x})\rangle \approx 0$ and 


$$
\left\langle u(x) u^{*}(\bar{x})\right\rangle=L\left(\frac{W_{0}}{\hbar v_{F}}\right)^{2}(1-2|x-\bar{x}| / L) .
$$

The prefactor can be related to the reflection coefficient of the wire. To see this we consider the Dyson equation to second order in the disorder,

$$
\begin{aligned}
\mathcal{G}\left(x, x^{\prime}\right) \simeq & \mathcal{G}_{0}\left(x, x^{\prime}\right)+\int_{0}^{L} d \chi \mathcal{G}_{0}(x, \chi) V(\chi) \mathcal{G}_{0}\left(\chi, x^{\prime}\right) \\
& +\int_{0}^{L} d \chi \int_{0}^{L} d \chi^{\prime} \mathcal{G}_{0}(x, \chi) V(\chi) \mathcal{G}_{0}\left(\chi, \chi^{\prime}\right) \\
& \times V\left(\chi^{\prime}\right) \mathcal{G}_{0}\left(\chi^{\prime}, x^{\prime}\right),
\end{aligned}
$$

and from the Fisher-Lee relation we notice that $\mathcal{R}=1-\mathcal{T}$ $=1-\left(\hbar v_{F}\right)^{2}|\mathcal{G}(L, 0)|^{2}$. It then follows that to second order in the disorder $\langle\mathcal{R}\rangle \simeq L\left(W_{0} / \hbar v_{F}\right)^{2}=L / l$ (the last equality follows from Appendix B) so that

$$
\left\langle u(x) u^{*}(\bar{x})\right\rangle=\langle\mathcal{R}\rangle(1-2|x-\bar{x}| / L)
$$

This means that

$$
\begin{aligned}
\left\langle\delta \Delta\left(x, x^{\prime}\right) \delta \Delta\left(\bar{x}, \bar{x}^{\prime}\right)\right\rangle= & \frac{\langle\mathcal{R}\rangle}{2}\left\{f\left(x^{\prime}-\bar{x}^{\prime}, x-\bar{x}\right)\right. \\
& +f\left(x-\bar{x}, x^{\prime}-\bar{x}^{\prime}\right)-f\left(x^{\prime}-\bar{x}, x-\bar{x}^{\prime}\right) \\
& \left.-f\left(x-\bar{x}^{\prime}, x^{\prime}-\bar{x}\right)\right\}
\end{aligned}
$$

where $f\left(x^{\prime}-\bar{x}^{\prime}, x-\bar{x}\right)=\left(1-2\left|x^{\prime}-\bar{x}^{\prime}\right| / L\right) \cos 2 k_{F}(x-\bar{x})$.

Out of the 16 terms only 4 terms of the form $\cos (x$ $-\bar{x}) \cos (y-\bar{y})$ are finite for long wires $k_{F} L \gg 1$ and we thus get

$$
\begin{aligned}
\left\langle\left[\delta G_{21}\right]^{2}\right\rangle_{\mathrm{uc}} \simeq & \mathcal{C}^{2}\left\langle\mathcal{R}_{1}\right\rangle\left\langle\mathcal{R}_{2}\right\rangle \int U_{12}(x, y) U_{12}\left(x^{\prime}, y^{\prime}\right) \\
& \times U_{12}(\bar{x}, \bar{y}) U_{12}\left(\bar{x}^{\prime}, \bar{y}^{\prime}\right) f\left(x-\bar{x}, x^{\prime}-\bar{x}^{\prime}\right) \\
& \times f\left(y-\bar{y}, y^{\prime}-\bar{y}^{\prime}\right) \\
= & \mathcal{C}^{2} \frac{1}{2}\left\langle\mathcal{R}_{1}\right\rangle\left\langle\mathcal{R}_{2}\right\rangle U_{12}^{2}\left(2 k_{F}\right) \widetilde{U}_{12}^{2}(0) .
\end{aligned}
$$

This corresponds to the estimate in Eq. (21c). Finally, the mean value is close to the value for ballistic wires and normalizing by Eq. (27) we get

$$
\frac{\left\langle\left[\delta G_{21}\right]^{2}\right\rangle_{\mathrm{uc}}^{1 / 2}}{G_{21}(\infty)} \simeq \frac{\left[2\left\langle\mathcal{R}_{1}\right\rangle\left\langle\mathcal{R}_{2}\right\rangle U_{12}^{2}\left(2 k_{F}\right) \widetilde{U}_{12}^{2}(0)\right]^{1 / 2}}{U_{12}^{2}\left(2 k_{F}\right)},
$$

where

$$
\begin{aligned}
\widetilde{U}_{12}^{2}(0) \equiv & \int_{0}^{L} \int_{0}^{L} \int_{0}^{L} \int_{0}^{L} d x_{1} d x_{2} d x_{1}^{\prime} d x_{2}^{\prime} \\
& \times U_{12}\left(x_{1}, x_{2}\right) U_{12}\left(x_{1}^{\prime}, x_{2}^{\prime}\right)\left(1-\frac{2\left|x_{1}-x_{1}^{\prime}\right|}{L}\right) \\
& \times\left(1-\frac{2\left|x_{2}-x_{2}^{\prime}\right|}{L}\right)
\end{aligned}
$$

and $\left\langle\mathcal{R}_{i}\right\rangle=L / l$. Equation (40) predicts the $1 / l$ dependence found in the qualitative discussion leading to Eq. (22). It follows that the relative magnitude of the fluctuations is of the order $\langle\mathcal{R}\rangle U_{12}(0) / U_{12}\left(2 k_{F}\right)$ and even though $\langle\mathcal{R}\rangle \ll 1$ for quasiballistic wires the effect of long-ranged interactions $U_{12}(0) / U_{12}\left(2 k_{F}\right) \gg 1$ can give rise to relative fluctuations of order unity, i.e., fluctuations comparable to the mean value. As we shall see in the next section, Eq. (40) is in excellent agreement with our numerical results.

\section{NUMERICAL RESULTS}

In this section we apply Eq. (15) to numerically evaluate the statistical properties of ensembles of different disorder configurations.

The numerical implementation was tested by first calculating the Landauer conductance $G_{i i}$, Eq. (17), for various geometries which can also be solved analytically. We have also tested our use of the Anderson model for disordered wires. By changing the ratio $l / L$ from the localized regime $(l / L \ll 1)$ to the delocalized regime $(l / L \gg 1)$ we have obtained distributions, mean values, and fluctuations for the Landauer conductance $G_{i i}$ which are in agreement with the analytical results of Abrikosov. ${ }^{27}$ We have also compared our lattice implementation, Eq. (15), to analytical results (see Appendix C) for drag between two ballistic wires. By making the grid sufficiently fine Eq. (15) is capable of reproducing the curves in Fig. 7 which are based on Eq. (C3) with the integrals evaluated numerically. Another test is the predicted peaking of $G_{21}$ at the onset of modes in either of the two wires ${ }^{14}$ and for systems with resonance transmission (peaks in $G_{i i}$ ); we find this to be borne out by our lattice implementation.

For the study of drag between disordered wires we consider quarter-filled bands $\left(\varepsilon_{F}=t\right)$ and wires with $N=100$ lattice points so that $k_{F} L=(\pi / 3) \times 100$. The separation is $k_{F} d=1$ and for simplicity we assume an unscreened Coulomb interaction

$$
\left\{U_{12}\right\}_{n n^{\prime}}=\frac{e^{2}}{4 \pi \epsilon_{0} \epsilon_{r} \sqrt{\left(n-n^{\prime}\right)^{2} a^{2}+d^{2}}} .
$$

For a discussion on how to include an effective screened interaction (e.g., within the random-phase approximation) see, e.g., Ref. 17 and references therein.

For the case $l=36 L$ (this corresponds to $W=\varepsilon_{F} / 10$ ) the disorder has as expected ${ }^{27}$ almost no effect on the Landauer conductance, i.e., $\left\langle G_{i i}\right\rangle \sim 2 e^{2} / h$ with vanishing fluctuations. However, for the transconductance the situation is very different. Panel (a) of Fig. 5 shows a typical histogram of 


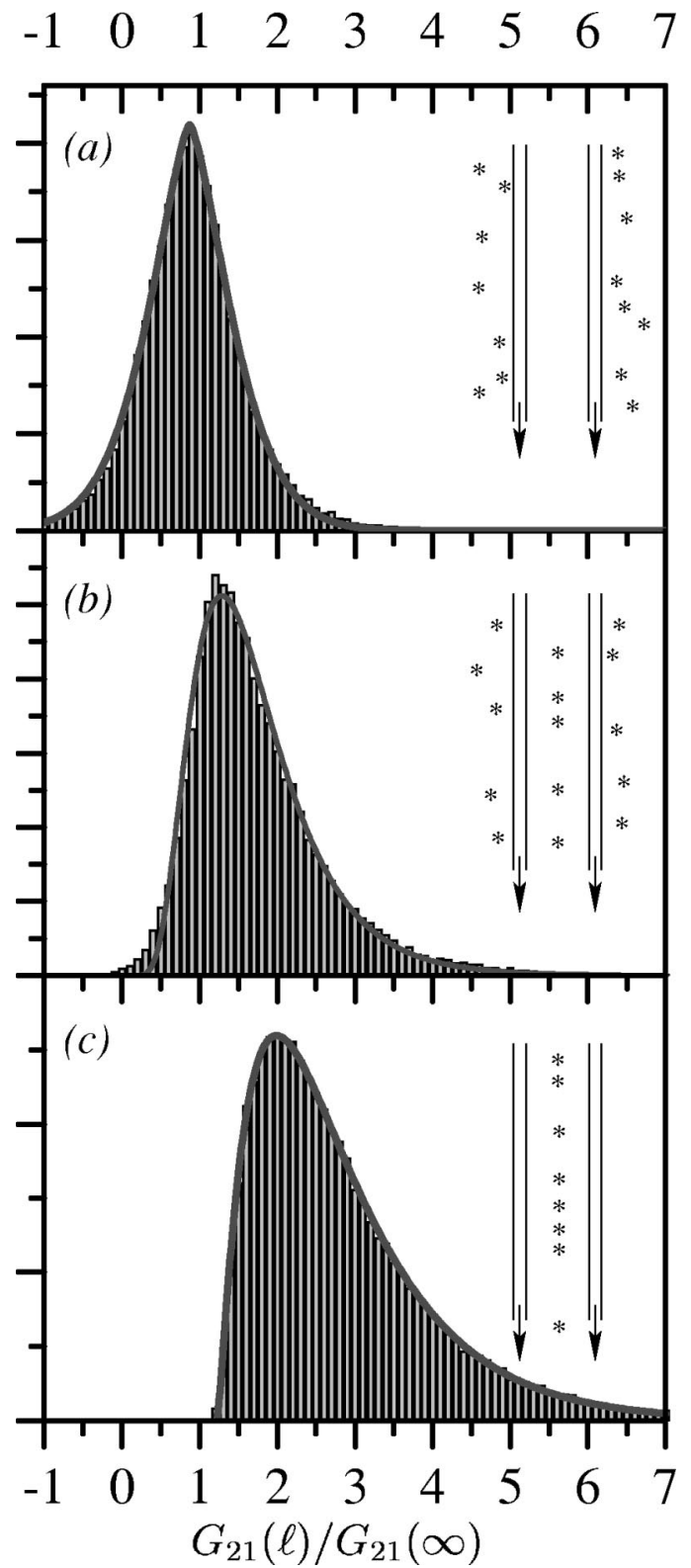

FIG. 5. Histograms for $G_{21}(l)$ normalized by the ballistic result $G_{21}(\infty)$. The histograms are based on $>10^{4}$ disorder configurations and for all three histograms the mean free path is $l=36 L$. Panel (a) is for the situation of mutually uncorrelated disorder, panel (b) for partly correlated disorder $\left(W_{\mathrm{c}}=W_{\mathrm{uc}}\right)$, and panel (c) is for mutually fully correlated disorder.

$G_{21}(l) / G_{21}(\infty)$ [where $G_{21}(\infty)$ is the result in the ballistic regime; see Appendix C] for $l=36 L$ in the case of uncorrelated disorder. Depending on the disorder configuration $G_{21}(l)$ can be either higher or lower than in the ballistic regime. The enhancement occurring for certain disorder configurations can be understood physically as follows. The lack of translational invariance allows forward scattering (transferred momentum $q \simeq 0$ ), which normally has little effect, to cause transitions between scattering states with opposite directions, thus contributing to the drag. We emphasize that the histogram peaks close to the ballistic value and not at zero drag; i.e., the mean drag is finite and positive. The variance

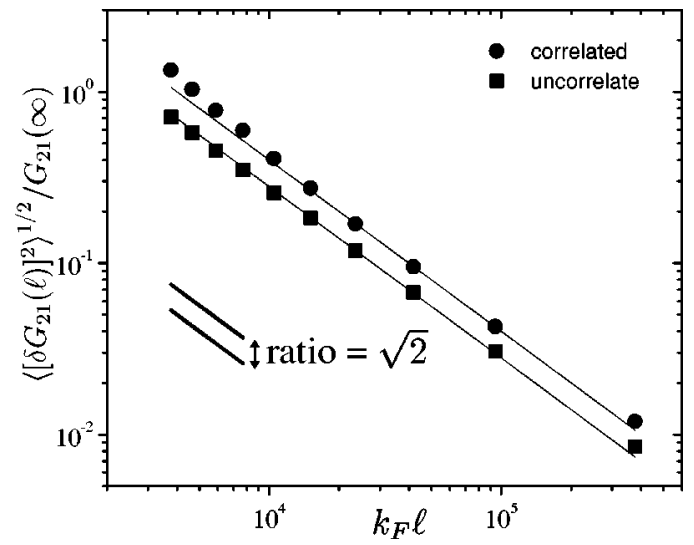

FIG. 6. Plot of fluctuations $\left\langle\left[\delta G_{21}(l)\right]^{2}\right\rangle^{1 / 2}$ as a function of the mean free path $k_{F} l$. In both cases the expected $1 / l$ behavior, Eq. (22), is borne out by the numerical calculations. The solid lines are Eq. (40) with no free parameters. The expected enhancement, Eq. (23), for correlated disorder by a factor of $\sqrt{2}$ compared to uncorrelated disorder is also confirmed by the numerical calculations.

is of the same order as the mean value so that sign reversal for some disorder realizations is possible. The latter is represented by the negative tail in the histogram. The sign of the drag is thus arbitrary in the sense that both positive and negative drag can be observed.

For the distribution of $G_{21}$ we find that $P_{\mathrm{uc}}(x)$ $\propto \exp \left[-|(x-\bar{x}) / \tilde{x}|^{\alpha}\right]$ with $\alpha \simeq 1.4$ fits surprisingly well to the data. However, this observation does not exclude the possibility that other functions may be fitted equally well. ${ }^{28}$ In fact we have performed these fits to histograms for $k_{F} l$ ranging from $10^{3}$ to $10^{5}$ with $k_{F} L$ in the range 100-300 and find indications that $\alpha \sim 1.4-1.5$ which by rescaling of $G_{21}$ makes it possible to let all histograms fall onto the same curve. However, the distribution is nonuniversal in the sense that it depends on the range of the interaction $U_{12}$.

For the same system parameters but now with fully correlated disorder we get a very different distribution as seen in panel (c) of Fig. 5. In this situation the mean transconductance is enhanced compared to the uncorrelated case. This confirms at least qualitatively the predictions for 2D systems of Ref. 18. The mean fluctuations are also enhanced (we return to the enhancement below). However, since the mean transconductance is enhanced by almost a factor of 2 compared to the ballistic limit this also means that there is no disorder configurations giving rise to negative drag. For partly correlated disorder, panel (b), the distribution is shifted to a higher mean value and the shape is also slightly changed with an increase in the magnitude of the fluctuations.

In Fig. 6 we show the dependence of the fluctuations on the mean free path $l$ which has the expected $1 / k_{F} l$ dependence; see Eq. (22). We also notice that correlated disorder gives rise to slightly larger fluctuations compared to uncorrelated disorder. In fact they are exactly enhanced by a factor of $\sqrt{2}$ as predicted; see Eq. (23).

\section{DISCUSSION AND CONCLUSION}

We have studied drag of Coulomb-coupled disordered mesoscopic 1D wires and developed a formalism for inves- 
tigating the statistical properties both analytically and numerically. In this work we have focused on the quasiballistic regime with $L \ll l$. For the ordinary Landauer conductance $G_{i i}$ this is experimentally a somewhat trivial regime since weak disorder has almost no effect and $\left\langle G_{i i}\right\rangle \sim 2 e^{2} / h$ with vanishing fluctuations. However, surprisingly this same regime (which can be achieved experimentally) offers rich physics when measuring transconductance between two Coulomb-coupled wires. In this work we report results of the statistical properties of the transconductance $G_{21}$ including its distribution function. Depending on the disorder configuration the transconductance can be either positive or negative and even though the mean value is close to the ballistic value the fluctuations can be of the same order of magnitude. This is fully explained by analytical calculations including the weak disorder perturbatively. The effect can be explained by a combination of disorder induced backscattering in the wires and forward scattering between the wires induced by Coulomb interaction.

We have also studied the recently proposed situation ${ }^{18}$ where the electrons in the two wires experience a common disorder potential. In this case we also find that even weak disorder gives rise to pronounced fluctuations and also a considerable enhancement of the mean transconductance.

The reported distribution functions have been successfully fitted by simple analytical expressions. Although there are no analytical predictions for the exact functional form, these initial results suggest that a more detailed analytical investigation could be rewarding.

Finally, let us note that while this work has focused on $L \ll l$, for multimode wires also the diffusive and localized regimes ${ }^{25,29}$ with $L>l$ seem to be a promising direction for future work. ${ }^{14}$

\section{ACKNOWLEDGMENTS}

We thank C. W. J. Beenakker, M. Brandbyge, and V. V. Cheianov for useful discussions. N.A.M. acknowledges financial support by "Ingeniørvidenskabelig Fond" and "G.A. Hagemanns Mindefond."

\section{APPENDIX A: FORMULATION IN TERMS OF SPECTRAL FUNCTIONS}

We start from Eq. (2) of Ref. 14. With a knowledge of the exact eigenstates of the isolated wires the expression points out a direct way of calculating the transconductance and it is possible to simplify this expression for ballistic wires where the eigenstates are known analytically; see Appendix C. However, in most cases the eigenstates are not known and it is useful to make a numerically more appropriate formulation in terms of the retarded lattice Green function. Using that the current matrix element can be written as

$$
\langle\alpha|\hat{J}(x)| \beta\rangle=\frac{\hbar}{2 m i_{\tilde{x} \rightarrow x}} \lim _{\tilde{x}}[\langle\alpha \mid x\rangle\langle\tilde{x} \mid \beta\rangle-\langle\alpha \mid \tilde{x}\rangle\langle x \mid \beta\rangle],
$$

and by introducing the factor $1=\int \mathrm{d} \varepsilon \delta\left(\varepsilon-\varepsilon_{\alpha}\right)$ into Eq. (2) of Ref. 14 we get the result in Eq. (3). In the second term we have used that the interchange $x^{\prime} \leftrightarrow x^{\prime \prime}$ corresponds to a sign change along with a complex conjugation.

\section{APPENDIX B: MEAN FREE PATH IN THE ANDERSON MODEL}

For a tight-binding chain with $N$ sites and no disorder we have eigenstates $\psi_{k}(n)=N^{-1 / 2} \exp (i k n a)$. We calculate the rate for forward scattering $(+)$ and backscattering $(-)$ from Fermi's golden rule

$$
\frac{1}{\tau_{ \pm}(k)}=\frac{2 \pi}{\hbar} \sum_{k^{\prime}}\left|\left\langle\psi_{k^{\prime}}|V| \psi_{k}\right\rangle\right|^{2} \delta\left(\varepsilon-\varepsilon^{\prime}\right) \frac{1 \pm k k^{\prime} /|k|\left|k^{\prime}\right|}{2}
$$

where $V$ is the disorder potential which is treated as a perturbation. For a chain with large $N$ we take the sum into an integral and since $\delta\left(\varepsilon-\varepsilon^{\prime}\right)=\left|\hbar v_{k}\right|^{-1}\left[\delta\left(k-k^{\prime}\right)+\delta(k\right.$ $\left.+k^{\prime}\right)$ ] we get

$$
\frac{1}{\tau_{ \pm}(k)}=\frac{a}{N \hbar^{2}\left|v_{k}\right|} \sum_{n m} e^{i(1 \mp 1) k(n-m) a} V_{n} V_{m}
$$

In the Anderson model ${ }^{24}$ the different sites are uncorrelated and $p\left(V_{n}\right)=\Theta\left(W / 2-V_{n}\right) / W$ which means that $\left\langle V_{n} V_{m}\right\rangle=\left\langle V_{n}^{2}\right\rangle \delta_{n m}=W^{2} / 12 \delta_{n m}$. The corresponding mean free path is given by $l(k)=v_{k} /\left\langle\tau_{ \pm}^{-1}(k)\right\rangle$ (forward scattering and backscattering give rise to the same result) and for $\varepsilon$ $=2 t(1-\cos k a)$ we get the result in Eq. (19) at the Fermi level. The result agrees with Ref. 25 except for a constant shift of the energy by $2 t$.

\section{APPENDIX C: BALLISTIC REGIME}

For ballistic wires the eigenstates are of the form $\psi_{k}(x)$ $=L^{-1 / 2} \exp (i k x)$ with $\varepsilon=\hbar^{2} k^{2} / 2 m$. The spectral function, Eq. (4), is then given by

$$
A_{\varepsilon}\left(x, x^{\prime}\right)=k(\varepsilon) \cos \left[k(\varepsilon)\left(x^{\prime}-x\right)\right] / \varepsilon .
$$

We consider the low-temperature limit $k T \ll \varepsilon_{F}$ where we can evaluate the spectral functions in Eq. (3) at the Fermi level so that

$$
\Delta_{i}\left(\omega, x^{\prime}, x^{\prime \prime}\right)=\frac{m}{2 \pi \hbar^{2}} \frac{\hbar \omega}{\varepsilon_{F}} \sin \left[2 k_{F}\left(x^{\prime}-x^{\prime \prime}\right)\right] .
$$

The $\omega$ integration in Eq. (2) can now be performed and using Eq. (14) we get

$$
\begin{aligned}
G_{21}= & \frac{e^{2}}{h} \frac{1}{12}\left(\frac{k T}{\varepsilon_{F}}\right)^{2}\left(\frac{2 m}{\hbar^{2}}\right)^{2} \\
& \times \int_{0}^{L} \int_{0}^{L} \int_{0}^{L} \int_{0}^{L} d x_{1}^{\prime} d x_{2}^{\prime} d x_{1}^{\prime \prime} d x_{2}^{\prime \prime} \sin \left[2 k_{F}\left(x_{1}^{\prime}-x_{1}^{\prime \prime}\right)\right] \\
& \times U_{12}\left(x_{1}^{\prime} ; x_{2}^{\prime}\right) U_{12}\left(x_{1}^{\prime \prime} ; x_{2}^{\prime \prime}\right) \sin \left[2 k_{F}\left(x_{2}^{\prime}-x_{2}^{\prime \prime}\right)\right] .
\end{aligned}
$$




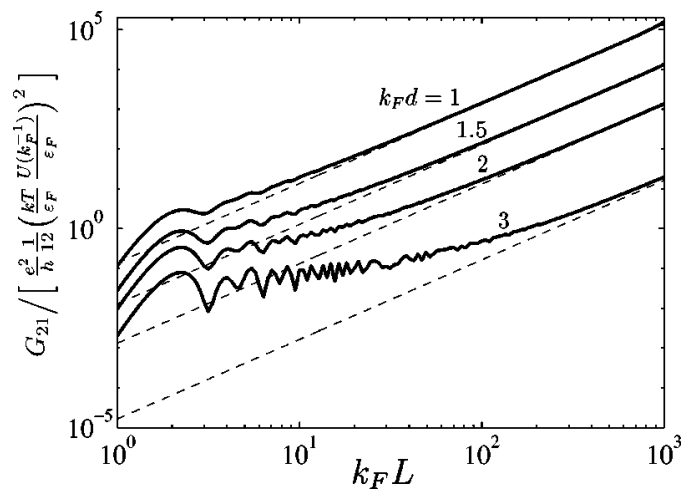

FIG. 7. Plot of $G_{21}$ for two ballistic wires, Eq. (C3), as a function of the length $k_{F} L$ for different values of the separation $k_{F} d$. The dashed curves show the asymptotic result, Eq. (C4).

In general the integrals have to be calculated numerically and only in the limit $k_{F} L \gg 1$ (the limit also studied in Ref. 5) we have the asymptotic result

$$
G_{21} \simeq \frac{e^{2}}{h} \frac{1}{6}\left(\frac{k T}{\varepsilon_{F}} \frac{U\left(k_{F}^{-1}\right)}{\varepsilon_{F}} k_{F} L K_{0}\left(2 k_{F} d\right)\right)^{2},
$$

where $K_{0}$ is a modified Bessel function of the second kind of order zero. ${ }^{30}$ Here, we have assumed unscreened Coulomb interaction $[$ see Eq. (41)] and introduced $U(r)$ $=e^{2} /\left(4 \pi \epsilon_{0} \epsilon_{r} r\right)$. Figure 7 shows a numerical evaluation of Eq. (C3) along with the asymptotic result, Eq. (C4).

\section{APPENDIX D: FORMULATION IN TERMS OF SCATTERING STATES}

We start from Eq. (4) in Ref. 14 using scattering states at the Fermi level as our basis: $\left\{\psi_{+}(x), \psi_{-}(x)\right\}$ where $+(-)$ is for a state incident from the left (right) lead. All matrix elements $I_{\alpha \beta}$ and $\rho_{\alpha \beta}$ can then be considered as elements of $2 \times 2$ matrices: $I$ and $\rho$. Due to current conservation, we are free to evaluate the current matrix element in the leads (outside the region with elastic scattering) which gives $I$ $=\left(v_{F} / L\right) J$ with

$$
J=\left(\tau^{3}-S^{\dagger} \tau^{3} S\right) / 2=\left(\begin{array}{cc}
\mathcal{T} & -\sqrt{\mathcal{R} T} e^{i(\phi-\theta)} \\
-\sqrt{\mathcal{R} T} e^{-i(\phi-\theta)} & -\mathcal{T}
\end{array}\right),
$$

where $\tau^{3}$ is the third Pauli matrix and

$$
S=\left(\begin{array}{cc}
r & t \\
t & r^{\prime}
\end{array}\right)=\left(\begin{array}{cc}
\sqrt{\mathcal{R}} e^{i \theta} & \sqrt{\mathcal{T}} e^{i \phi} \\
\sqrt{\mathcal{T}} e^{i \phi} & -\sqrt{\mathcal{R}} e^{i(2 \phi-\theta)}
\end{array}\right)
$$

is the usual unitary scattering matrix in the presence of timereversal symmetry $\left(S S^{\dagger}=\hat{1}\right.$ and $\left.S=S^{T}\right)$. In the second equality the scattering probabilities $\mathcal{T}=|t|^{2}$ and $\mathcal{R}=1-\mathcal{T}$ $=|r|^{2}=\left|r^{\prime}\right|^{2}$ have been introduced. Equation (4) in Ref. 14 can now be written as

$$
\Delta\left(\omega, x, x^{\prime}\right)=\pi^{2} \hbar \omega \frac{\hbar v_{F}}{L} \operatorname{Tr}\left\{J\left[\rho(x) ; \rho\left(x^{\prime}\right)\right]\right\},
$$

where $[A ; B]=A B-B A$ is a commutator with $A$ and $B$ being matrices. Next we employ a unitary transformation

$$
\mathcal{U}=\left(\begin{array}{cc}
u & -v \\
v^{*} & u^{*}
\end{array}\right)
$$

with $|u|^{2}+|v|^{2}=1$, which satisfies $\mathcal{U} J \mathcal{U}^{\dagger}=\sqrt{\mathcal{T}} \tau^{3}$ by choosing $|u|^{2}=\frac{1}{2}(1+\sqrt{\mathcal{T}}), \quad|v|^{2}=\frac{1}{2}(1-\sqrt{\mathcal{T}}), \quad$ and $\quad v u^{*}$ $=\frac{1}{2} \sqrt{\mathcal{R}} e^{i(\phi-\theta)}$ (in a concrete calculation it can be useful to use the freedom to choose the phases as $v=|v| e^{i(\phi-\theta) / 2}$ and $\left.u=|u| e^{-i(\phi-\theta) / 2}\right)$. It is then easy to obtain the very compact result

$$
\Delta\left(\omega, x, x^{\prime}\right)=4 \pi^{2} \hbar \omega \frac{\hbar v_{F}}{L} \sqrt{\mathcal{T}} i \operatorname{Im}\left\{\tilde{\rho}_{+-}(x) \tilde{\rho}_{-+}\left(x^{\prime}\right)\right\},
$$

where in the new basis $\tilde{\rho}(x)=\mathcal{U}^{\dagger} \rho(x) \mathcal{U}$. With the choice of relative phase mentioned above we have in particular

$$
\begin{aligned}
\tilde{\rho}_{+-}(x)= & \frac{e^{i(\phi-\theta)}}{2}\left[\sqrt{\mathcal{R}}\left[\rho_{--}(x)-\rho_{++}(x)\right]\right. \\
& \left.+(1+\sqrt{\mathcal{T}}) \rho_{+-}(x)-(1-\sqrt{\mathcal{T}}) \rho_{+-}^{*}(x)\right] .
\end{aligned}
$$

${ }^{7}$ K. Flensberg, Phys. Rev. Lett. 81, 184 (1998).

${ }^{8}$ V.V. Ponomarenko and D.V. Averin, Phys. Rev. Lett. 85, 4928 (2000).

${ }^{9}$ A. Komnik and R. Egger, Phys. Rev. Lett. 80, 2881 (1998).

${ }^{10}$ A. Komnik and R. Egger, Eur. Phys. J. B 19, 271 (2001).

${ }^{11}$ R. Klesse and A. Stern, Phys. Rev. B 62, 16912 (2000).

${ }^{12}$ S. Feng, C. Kane, P.A. Lee, and A.D. Stone, Phys. Rev. Lett. 61, 834 (1988).

${ }^{13}$ B.N. Narozhny and I.L. Aleiner, Phys. Rev. Lett. 84, 5383 (2000).

${ }^{14}$ N.A. Mortensen, K. Flensberg, and A.-P. Jauho, Phys. Rev. Lett. 86, 1841 (2001). 
${ }^{15}$ N. A. Mortensen, K. Flensberg, and A.-P. Jauho, cond-mat/ 0108203, Phys. Scr. (to be published).

${ }^{16}$ A. Kamenev and Y. Oreg, Phys. Rev. B 52, 7516 (1995).

${ }^{17}$ K. Flensberg, B.Y.-K. Hu, A.-P. Jauho, and J.M. Kinaret, Phys. Rev. B 52, 14761 (1995).

${ }^{18}$ I.V. Gornyi, A.G. Yashenkin, and D.V. Khveshchenko, Phys. Rev. Lett. 83, 152 (1999).

${ }^{19}$ G. D. Mahan, Many-Particle Physics (Plenum Press, New York, 1990).

${ }^{20}$ H.U. Baranger and A.D. Stone, Phys. Rev. B 40, 8169 (1989).

${ }^{21}$ J.U. Nöckel, A.D. Stone, and H.U. Baranger, Phys. Rev. B 48, 17569 (1993).

${ }^{22}$ S. Datta, Electronic Transport in Mesoscopic Systems (Cambridge
University Press, Cambridge, England, 1995).

${ }^{23}$ H.U. Baranger, D.P. DiVincenzo, R.A. Jalabert, and A.D. Stone, Phys. Rev. B 44, 10637 (1991).

${ }^{24}$ P.W. Anderson, Phys. Rev. 109, 1492 (1958).

${ }^{25}$ B. Kramer and A. MacKinnon, Rep. Prog. Phys. 56, 1469 (1993).

${ }^{26}$ See, e.g., E. Merzbacher, Quantum Mechanics (Wiley, New York, 1970).

${ }^{27}$ A.A. Abrikosov, Solid State Commun. 37, 997 (1981).

${ }^{28}$ V. V. Cheianov (private communication).

${ }^{29}$ C.W.J. Beenakker, Rev. Mod. Phys. 69, 731 (1997).

${ }^{30}$ For the integration see Eq. (3.754.2) in I. S. Gradshteyn and I. M. Ryzhik, Table of Integrals, Series, and Products (Academic Press, New York, 1980). 